Submitted to Ap.J.;revised October 19, 2001

\title{
How Mira Variables Change Visual Light by a Thousand-fold
}

\author{
M. J. Reid and J. E. Goldston \\ Harvard-Smithsonian Center for Astrophysics, 60 Garden Street, Cambridge, MA 02138 \\ reid@cfa.harvard.edu, jgoldston@cfa.harvard.edu
}

\begin{abstract}
Mira variables change visual light by up to 8 magnitudes over their roughly yearly cycle. Here we present a simple explanation for the extremely large amplitudes of light curves of oxygen-rich Mira variables. Metallic oxides, such as $\mathrm{TiO}$, form throughout the stellar atmosphere as the star cools when approaching minimum light. When this happens, the visual light can be almost completely absorbed at large radii, extending the visual photosphere to nearly twice its nominal size. At these large radii, temperatures can fall to $\approx 1400 \mathrm{~K}$ and essentially all of the star's radiation emerges in the infrared. Since almost no optical light is emitted at these low temperatures, Mira variables can decrease their visual light by more than a thousand-fold and almost "disappear" to the human eye.
\end{abstract}

Subject headings: stars: late-type, AGB and post-AGB, variables, atmospheresmolecular processes

\section{Introduction}

Mira variables have long fascinated both amateur and professional astronomers. In the late 1500s, European and Asian astronomers noted the "disappearance" of a star, most likely o Ceti, over a period of months. While this star dimmed dramatically, it did not really disappear and, in the early 1600s, Fabricus and Holwarda recognized that $o$ Ceti was a variable star (Hoffleit 1997). Because of the extreme changes of its brightness, o Ceti was called Mira (or "marvelous one"). Indeed, Mira variables are marvelous; some regularly change visual luminosity by $\approx 8$ magnitudes, or factors of more than 1000 , over periods of hundreds of days (Merrill 1940). 
Mira variables are evolved red giants with extraordinarily large sizes; a typical Mira has a radius of $\approx 2 \mathrm{AU}$ (e.g., Pease 1931; Labeyrie et al. 1977; Lopez et al. 1997; Tej et al. 1999). Mira variables are thought to pulsate, owing to opacity from the variable ionization of hydrogen and helium below the stellar surface (e.g., Keeley 1970). These pulsations emerge as shock waves and propagate upwards into the lower photosphere near maximum visible light (e.g., Slutz 1976). A Mira variable can have a very complex, dynamic atmosphere, and much theoretical and computational work has been expended attempting to understand the physical processes involved (e.g., Wood 1979; Bertschinger \& Chavalier 1985; Bowen 1988; Bessel et al. 1989; Plez, Brett \& Nordlund 1992; Höfner et al. 1998; Woitke et al. 1999; Helling, Winters \& Sedlmayr 2000). Because Miras are so large, their surface temperatures are low and many molecules form in their atmospheres. At distances of a few stellar radii, temperatures fall even lower, which allows the formation of silicate or graphite "dust." Once dust grains form, radiation pressure rapidly disperses them into interstellar space, enriching the environment with heavy elements. The ability of red giant stars to form and disperse circumstellar material is crucial to the life-cycle of stars and for life as we know it. Thus, the study of the workings of the cool atmospheres of such stars has great significance to astrophysics.

Nearly 70 year ago, Pettit \& Nicholson (1933) showed that Mira variables have temperature variations of $\approx 30 \%$ and total luminosity variations of factors of $\approx 2$ over their roughly yearly cycle. The luminosity, $L$, the effective temperature, $T_{\text {eff }}$, and the radius, $R$, of a star are related by $L=\sigma T_{\text {eff }}^{4} \pi R^{2}$, where $\sigma$ is the Stephan-Boltzmann constant. Using this relation, measurements of $L$ and $T_{\text {eff }}$ give an estimate of $R$, and radii of Miras determined in this manner indicate size changes of $\approx 40 \%$.

The observed visual (Mattei 2001) and infrared light (Lockwood \& Wing 1971) curves of $\chi$ Cygni, a bright oxygen-rich Mira variable, are presented in Fig. 1 as green and red crosses, respectively. Plotted with solid lines are the expected flux density as a function of time for a time-variable black-body model based on changes in temperature and size as determined by Pettit \& Nicholson (1933). The infrared model falls only about 1 magnitude dimmer than the data and has approximately the correct amplitude. Thus, by adjusting the stellar size, this model could fit the infrared light curve well. However, the visible curve (green) starts at maximum light about 2 magnitudes too bright, and the amplitude is about 4 magnitudes too small. This is a general problem for Mira variables: the measured temperature and radius changes, determined mostly from infrared data, predict only about 3 to 4 magnitudes of visual light variation, whereas up to 8 magnitudes are sometimes observed.

Good fits to the observed light curves cannot be obtained by simply changing the variation of the temperature and radius of a stellar black-body. If the black-body temperatures 
are allowed to decrease considerably below those indicated by infrared data, one can generate a very large visual amplitude and reproduce the visual magnitude at minimum light. For example, in Fig. 1 we show light curves (dashed lines) for a stellar black-body whose temperature varies from $2300 \mathrm{~K}$ at maximum light to $1300 \mathrm{~K}$ at minimum light. While the visual light curve can be successfully modeled in this manner, the model for the infrared light curve degrades considerably. The problem is that time varying black-body models cannot simultaneously fit the optical and infrared light curves of a star like $\chi$ Cygni.

Perhaps one could explain Mira light curves through the effects of a strong opacity source that varies considerably with wave-band. Astronomers have long suspected that molecular absorption in the atmospheres of Mira variables contributed to the large change in visual luminosity (e.g., Pettit \& Nicholson 1933; Smak 1964; Celis 1978). However, to our knowledge, this problem has never been directly addressed. In this paper, we present an explanation of how the formation of metallic oxides (notably $\mathrm{TiO}$ ), as the star periodically cools, can solve the problem of Mira light curves.

\section{Basic Physical Explanation}

The fact that Mira variables change infrared (and total) luminosity by only about 1 magnitude suggests that the star is not undergoing exotic changes. Where then should one look to explain the dramatic optical variations? Consider a star with an average temperature of $2200 \mathrm{~K}$ and a radius of $2 \mathrm{AU}$. At a distance of 100 parsecs, this star would have visual magnitude, $m_{v}$, of $+4.5 \mathrm{mag}$. Varying the radius of such a star by $\pm 20 \%$ (from 2.4 to 1.6 $\mathrm{AU})$ changes $m_{v}$ by only $\pm 0.4 \mathrm{mag}$. Clearly, size changes are insufficient to explain the large observed swings in visual light. On the other hand, varying the temperature of the star by only $\pm 15 \%$ (from $2500 \mathrm{~K}$ to $1900 \mathrm{~K}$ ) strongly affects visible light — changing it by $3.4 \mathrm{mag}$. While this is inadequate to explain the large changes in the visual light of many Miras, it does suggest that the explanation comes predominantly through the effects of temperature variations.

The emission from a Mira variable with an 8 magnitude amplitude could be explained if, at minimum light, the radiation comes from material at about $1400 \mathrm{~K}$. How far above the stellar surface ${ }^{1}$ does this temperature occur? If, for example, the temperature decreases with radius as $r^{-1 / 2}$, then a star with a minimum surface temperature of $1900 \mathrm{~K}$ will experience temperatures near $1400 \mathrm{~K}$ at about 1.8 stellar radii $\left(R_{*}\right)$. Thus, the problem of explaining

\footnotetext{
${ }^{1}$ Throughout this paper we use the terms stellar surface, stellar size, and stellar radius to mean those quantities measured at infrared wavelengths in line-free regions.
} 
the large amplitude of Mira variables may be reduced to finding a source of visual opacity sufficient to make the star appear $80 \%$ larger than the stellar size at minimum light, while remaining close the stellar size at maximum light. In order to accomplish this, the opacity in the stellar atmosphere should have the following characteristics: 1) The opacity must increase significantly as the temperature decreases from about 1900 to $1400 \mathrm{~K}$. Were, for example, the opacity to remain high throughout the stellar cycle, the amplitude of the variations would only approach about 5 magnitudes. 2) The visual opacity must be great enough to achieve optical depths of $\sim 1$ for the low densities expected in the stellar atmosphere at $r \approx 2 R_{*}$.

It has long been known that oxygen-rich (M- and S-type) Miras have strong metallic oxide absorption bands across the visible spectrum, with titanium oxide (TiO) typically providing the dominant absorption (Merrill 1940). In the extended photosphere of an oxygen rich Mira variable, the chemical reaction of $\mathrm{Ti}+\mathrm{O} \rightleftharpoons \mathrm{TiO}$ proceeds from predominantly atomic to molecular phases for temperatures between about 2000 and $1600 \mathrm{~K}$ for total densities of $10^{-10}$ and $10^{-14} \mathrm{gm} \mathrm{cm}^{-3}$, respectively. So as the star cools when approaching minimum light, titanium can transition from mostly atomic to molecular form in the extended atmosphere of a Mira variable. Once TiO forms, it is extremely efficient at absorbing optical photons. This is why some sun screen products use titanium (or other metallic) oxides.

The implications for a star that naturally forms sun screen in its atmosphere can be dramatic. The optical depth to molecular absorption can be written as follows: $\tau=\int n_{m} \alpha d \ell$, where $n_{m}$ is the molecular density, $\alpha$ is the absorption cross-section, and $d \ell$ is path length along our line-of-sight. Can one achieve $\tau \sim 1$ in the extended photosphere owing to $\mathrm{TiO}$ absorption? For an approximate estimate of opacity, assume a characteristic path length of $\ell \sim 10^{13} \mathrm{~cm}$ and a TiO cross-section, averaged over the visual band, of $\alpha \sim 10^{-17} \mathrm{~cm}^{2}$ (Jorgensen 1994). Then unit optical depth requires a characteristic $\mathrm{TiO}$ number density of $n_{m}=10^{4} \mathrm{~cm}^{-3}$. For solar abundances, this corresponds to a total mass density of $\sim 10^{-13} \mathrm{gm} \mathrm{cm}^{-3}$. Such a mass density is reasonable in the extended stellar photosphere of a Mira variable. Thus, near minimum light $\mathrm{TiO}$ (and other metallic oxides) can absorb most of the optical light and produce a "visual surface" far above the true stellar surface.

The change in the visual appearance of a Mira between maximum and minimum visible light is schematically represented in Fig. 2. The left-hand panel of the figure represents the star near maximum visual light $(\phi=0)$; one can see through the extended atmosphere, in which metals such as Ti are predominantly in atomic form, almost down to the stellar surface. (Actually, even though the fraction of Ti in molecular form is very low at temperatures above $2000 \mathrm{~K}$, a reasonable amount of $\mathrm{TiO}$ is found near the stellar surface, owing to the extremely high densities encountered there.) The right-hand panel of Fig. 2 represents the star near minimum light $(\phi \approx 0.5)$; here the temperature of the star has declined and $\mathrm{TiO}$ is abundant 
in the extended photosphere. At this point, one can only "see" down to a radius of $\approx 1.8 R_{*}$. When this happens the visual intensity drops dramatically, which can explain why Mira variables change visual light by factors exceeding 1000.

\section{Model Light Curves}

It is exceedingly difficult, and probably beyond current capabilities, to model precisely all of the physical and chemical processes in the atmosphere of a Mira variable. However, in order to validate the basic physical explanation for Mira light curves, we need only approximate or representative light curves. This can be accomplished provided the temperature and density as a function radius and optical phase is available. We found four papers in the recent literature that contain time-dependent models of oxygen-rich Mira variables and for which temperature and density profiles are plotted for maximum and minimum visual light (Bertschinger \& Chavalier 1985, Fig. 13; Bowen 1988, Fig. 6; Bessel et al. 1989, Figs. 2 \& 4; Höfner et al. 1998, Fig. 9). These profiles were digitized and used as input physical models for calculations of apparent visual magnitudes. We note that there are very large differences in the temperature and density profiles among these models.

We used the following procedure to calculate apparent visual magnitudes. At each radius in the extended photosphere, we evaluated the chemical reaction for forming molecular TiO, assuming solar abundance ratios $\left([\mathrm{Ti}] /[\mathrm{H}]=9.8 \times 10^{-8}\right.$ and $\left.[\mathrm{O}] /[\mathrm{H}]=8.5 \times 10^{-4}\right)$, a TiO binding energy of $-6.9 \mathrm{eV}$, and chemical equilibrium. Since most of the oxygen is probably bound in $\mathrm{CO}$ and other molecules, we assumed that only about $1 \%$ of oxygen is available to bond to titanium. The absorption cross-section of $\mathrm{TiO}$ as a function of wavelength was estimated, based on calculations provided by R. Kurucz, for a range of temperatures and pressures appropriate for the conditions in the outer atmosphere of a Mira variable. The dependence of the cross-sections on pressure is insignificant, but the dependence on temperature is noticeable, and we interpolated the cross-sections in temperature between values calculated at 2200 and $1400 \mathrm{~K}$.

We calculated numerically the transfer of radiation through the atmosphere using a method similar to that described by Reid \& Menten (1997). At a given wavelength, we integrated the intensity contribution at each point along our line-of-sight toward the star, diminished by absorption along the path to the observer. This integration was repeated for different stellar impact parameters, and a monochromatic flux density was determined by summing the integrated intensity times the solid angle of an annulus appropriate for each impact parameter. Next we repeated the integrations over a range of wavelengths to sample the entire optical spectrum. Finally, the total flux density was obtained by summing over 
all wavelengths, accounting for the transmission characteristics of a visual filter (Johnson 1952). In this manner, we generated an apparent visual magnitude, $m_{v}$, for a given stellar phase.

The calculated apparent visual magnitudes at maximum light, using the published temperature and density profiles from the four papers cited above, spanned a range of about 10 magnitudes. The model of Bowen (1988) returned $m_{v}=6$, which closely matched the $\chi$ Cygni data at maximum light (assuming a distance of $136 \mathrm{pc}$; see discussion in next section). Other models typically yielded visual magnitudes a few magnitudes brighter. At minimum light, the model of Bowen resulted in $m_{v}=9$, about 4.5 magnitudes too bright. The differences in visual light between maximum and minimum for most of the models were only about 3 magnitudes, and for two of the four models the light at minimum exceeded that at maximum. Thus, these published temperature and density profiles cannot explain the visual light curve of a large amplitude Mira variable like $\chi$ Cygni. This should not, perhaps, come as a surprise. The visual light is only a small fraction of the total light from the star, and thus it is relatively unimportant in determining the physical and dynamical conditions in the atmospheric models. Since the published models have not been optimized to fit visual light, it is not surprising that current models fail in this regard. However, the visual light is a sensitive probe of physical conditions in the atmosphere of a Mira variable, and we hope that future models would be optimized to fit visual light curves.

Since published models seem inadequate for our purposes, we constructed a simple hydrostatic model for the stellar atmosphere and evaluated the transfer of radiation numerically as described above. This model is computationally manageable and its parameters are easily adjusted to attempt to fit light curves. The model assumes a star whose surface temperature and radius vary out of phase as follows:

$$
\begin{array}{r}
T_{*}=T_{0}+\Delta T \cos (\phi), \\
R_{*}=R_{0}+\Delta R \cos (\phi+\pi),
\end{array}
$$

where $\phi$ is the visual stellar phase. The phase shift of $\pi$ in Eq. (1b), forces the radius to be a minimum when the temperature is maximum (Pettit \& Nicholson 1933). This star is "embedded" in an extended photosphere. Following Reid \& Menten (1997), we assume that temperature as a function of radius, $T(r)$, in the extended photosphere decreases as follows:

$$
T(r)^{4}=T_{*}^{4}\left(1-\sqrt{1-\left(R_{*} / r\right)^{2}}\right) .
$$

This temperature profile decreases approximately as $r^{-1 / 2}$ (for $r \geq 1.2 R_{*}$ ) and, for hydrostatic equilibrium, results in a density profile, $n(r)$, decreasing with radius given by

$$
n(r) \approx n_{*} e^{-C\left(1-\left(R_{*} / r\right)^{1 / 2}\right)}
$$


In Eq. (3), $C=2 G M_{e f f} m_{H} / k T_{*} R_{*}, M_{e f f}$ is the effective mass of the star, $m_{H}$ is the hydrogen mass, $k$ is Boltzmann's constant, and $G$ is the gravitational constant. Examples of density and temperature profiles (for parameters given in $\S 4$ ) are shown in Fig. 3.

\section{Observed and Modeled Light Curves for $\chi$ Cygni}

We chose to model the star $\chi$ Cygni because 1) it displays a very large, consistent, change in visual light, and 2) excellent visual (Mattei 2001) and infrared (Lockwood \& Wing 1971) light curves are available. We adopted a distance to $\chi$ Cygni of $136 \mathrm{pc}$, based on the period-luminosity relation of Feast et al. (1989) and the photometry of Haniff, Scholz \& Tuthill (1995), and an effective stellar mass of $1 M_{\odot}$. We modeled the star as described in $\S 3$, adjusting the parameters of the model to fit the optical and infrared light curves. A good fit to the light curves of $\chi$ Cygni was obtained for a mean temperature and radius of $T_{0}=2200 \mathrm{~K}$ and $R_{0}=2.1 \mathrm{AU}$, respectively, for the central star. Changes in temperature, $\Delta T$, and radius, $\Delta R$, over the stellar cycle were set to $\pm 20 \%$, and a visual extinction of 0.6 mag was used. The total mass in the extended atmosphere was a held constant over the stellar cycle by adjusting the density parameter $n_{*}$.

We present the results of the radiative transfer calculations in Fig. 4. In addition to the visual light curve, we also required the model to reproduce the infrared light curve at a wavelength of $1.04 \mu \mathrm{m}$. At this wavelength, molecular opacity is very low (Lockwood \& Wing 1971), and the infrared radiation comes from the stellar surface. As one can see, this model successfully reproduces both the small variation in the infrared (where TiO opacity is low) and the extremely large changes in visual light. This demonstrates that, for reasonable physical parameters, one can explain the "marvelous" behavior of a Mira variable as outlined in $\S 2$.

Reid \& Menten (1997) measured the radio continuum emission from a sample of Mira variables and found that the radio emission comes from $\approx 2 R_{*}$. This is the same region of the extended photosphere that is optically thick at visual wavelengths, owing to molecular opacity, near minimum light. Since the radio opacity is from an entirely different source $\left(\mathrm{H}^{-}\right.$ free-free interactions) than the optical opacity, one can check how well our simple model predicts photospheric emission at radio wavelengths. Thus, we followed the procedures described by Reid \& Menten and calculated the emission at a wavelength of $3.6 \mathrm{~cm}$ that would result from the model for the optical and infrared emission described above. The predicted radio emission from the model of $\chi$ Cygni is also shown in Fig. 4. While a complete radio "light curve" is not yet available for $\chi$ Cygni, there are two measures of its $3.6 \mathrm{~cm}$ wavelength 
emission made with the $\mathrm{NRAO}^{2}$ Very Large Array: $0.27 \pm 0.04 \mathrm{mJy}$ at $\phi=0.21$ (Reid \& Menten) and $0.19 \pm 0.04$ mJy at $\phi=0.43$ (Reid, Menten \& Goldston 2001). We converted these flux densities to magnitude units and plot them in Fig. 4. The model, adjusted only to optical and infrared data, predicts the strength of the radio emission reasonably well. Also, the model predicts only a small variation of the radio flux density over the stellar cycle, which is consistent with that observed for other Mira variables (o Ceti, R Leo, and W Hya) where better phase coverage exists.

We have shown that a simple model can successfully fit the optical, infrared, and radio light curves of $\chi$ Cygni. However, the temperature and density profiles of Mira variables are likely far more complex. So, why is our simple model so successful? One might be concerned that the model fits the data well only because it has many adjustable parameters. Indeed, the model has 9 parameters. However, all of the parameters are physical ones, required by the problem. The stellar distance comes from Feast et al. (1989) and the stellar mass was arbitrarily set at $1 \mathrm{M}_{\odot}$; neither were adjusted. The two stellar temperature and radius parameters are constrained mostly by the infrared light curves. The visual extinction only shifts the entire light curve up or down; the value adopted is small and not important. This leaves only two parameters that can be used to fit the visual light curve: the oxygen depletion and a single atmospheric density parameter $\left(n_{*}\right)$. So, the good fit of the model visual light curve to the observed data cannot be a result of over-parameterization.

How sensitive is the model visual light curve to the precise nature of the temperature and density profiles? In order to investigate this question, we changed the density profile from an exponential form, appropriate for a hydrostatic atmosphere, to a power-law form, appropriate for a smoothly expanding atmosphere. Using temperature and density profiles proportional to $r^{-1 / 2}$ and $r^{-5}$, respectively (see Fig. 3), we were able to reproduce the large amplitude of the visual light curve of $\chi$ Cygni nearly as well as the exponential model, while also obtaining satisfactory agreement with the infrared and radio data. This suggests that the model light curves are somewhat robust to changes in the forms of the temperature and density profiles. Details of the variation of temperature and density with radius do not strongly affect the amplitude of the visual light curve, provided that sufficient opacity occurs near minimum light at radii where the temperature falls to a low value as discussed in $\S 2$.

\footnotetext{
${ }^{2}$ NRAO is a facility of the National Science Foundation operated under cooperative agreement by Associated Universities, Inc.
} 


\section{Comments and Caveats}

In some previous attempts to explain the phenomenon of Mira variables, the effects of $\mathrm{TiO}$ absorption have been considered as "extinction" - that is simply absorbing the stellar light. However, this explanation is incomplete, because the medium containing the absorbing material also re-emits light. Consider, for example, the Sun. The solar photosphere has a strong opacity source from the reaction $\mathrm{H}^{-} \rightarrow \mathrm{H}+\mathrm{e}^{-}$(Wildt 1939); indeed, essentially all visible Sun light comes from the formation of $\mathrm{H}^{-}$. However, since density falls steeply with radius in the Sun, the region of high opacity is confined to a very small range of radii. Across this region, the temperature changes only slightly, and the visible light from the Sun departs only marginally from a blackbody at a single temperature. Thus, strong absorption of light in a stellar photosphere is not alone sufficient to change the visual intensity markedly. The absorption must happen over a large range in radius, where the temperature changes significantly, if it is to dramatically change the visual intensity. This occurs in Mira variables, owing to molecular (e.g, TiO) absorption, but it does not occur in the Sun, even though there is equally strong $\mathrm{H}^{-}$absorption.

Our simple model cannot explain all details of the wide variety of light curves observed. Some Miras exhibit asymmetric light curves, which decay slowly and rise rapidly toward maximum light; others display secondary "humps" in their curves. Also, Mira variables are not perfectly spherical - some are elongated by $10 \%$ or more (Karovska et al. 1991). Photospheric shocks and mass outflow generally tend to soften the density profile (i.e., increase the density at larger radii) above that obtained in pure hydrostatic equilibrium. On the other hand, there is clear evidence that Mira atmospheres are not smoothly expanding. Regions of large-scale infall are indicated by red-shifted absorption lines (Reid 1976; Reid \& Dickinson 1976; Ferlet \& Gillet 1984) and they have been directly observed in the proper motions of some $\mathrm{SiO}$ masers (Diamond \& Kemball 1999). Thus, the combination of outflowing and infalling regions, perhaps better described as convective motions, when averaged over the entire atmosphere, might result in an average density profile that deviates less from a hydrostatic profile than expected from pure outflow. This needs to be studied. Still, explaining many details of Mira emissions requires more sophisticated modeling. The simple hydrostatic model used here was chosen for computational ease, since this paper is concerned with explaining the basic physical reasons for the large amplitude of the visual light curve and not with constructing a detailed atmospheric model. Ultimately, full dynamical models should be applied to this problem; these models should be constrained by observed light curves, in addition to observed spectra and measured sizes.

Our simplifying assumption, that the visual opacity is dominated by $\mathrm{TiO}$, is only a first approximation. Other sources contribute to the overall opacity. For example, stars of 
spectral class $\mathrm{S}$ exhibit strong bands from $\mathrm{ZrO}$. Indeed, $\chi$ Cygni has been variously classified as an M-type (strong $\mathrm{TiO}$ bands) and an S-type (strong $\mathrm{ZrO}$ bands in addition to $\mathrm{TiO}$ ) by different observers at different epochs. Including sources of opacity beyond $\mathrm{TiO}$ would decrease somewhat the required total density to achieve similar total opacity. This would further enhance the effects leading to the large amplitude of the visual light curves and make our explanation for these light curves more robust. Some carbon-rich Mira variables of spectral class $\mathrm{N}$ and $\mathrm{R}$ are also known to have large amplitude light curves (Merrill 1940). For these stars, essentially all oxygen is in $\mathrm{CO}$ and metallic oxides, such as TiO, cannot form abundantly. Thus, the explanation for the behavior of the light curves of carbon-rich Mira variables probably involves strong opacity from carbon-bearing molecules such as $\mathrm{C}_{2}$ and CN (Reid \& Goldston 2002). Finally, dust can form at temperatures below $\sim 1500-1200 \mathrm{~K}$ in carbon or oxygen rich Miras. Indeed, dust shells are directly observed in some Miras, usually at radii greater than about $3 R_{*}$ (Danchi et al. 1994), and these can also contribute to the extinction of optical light (e.g., Winters et al. 1994).

In conclusion, we mention that strong evidence for our basic picture comes from interferometric observations at visible and radio wavelengths. Interferometric observations in the visible show large changes in the apparent size of the star for wavelengths in and out of $\mathrm{TiO}$ absorption bands (Labeyrie et al. 1977; Tuthill, Haniff \& Baldwin 1999; Young et al. 2000). For example, imaging observations made with the Palomar 5-m telescope clearly show a "shell" of high opacity at $\approx 2 R_{*}$ in a strong TiO absorption band (Haniff et al. 1992). Independent observations at radio wavelengths (Reid \& Menten 1997) directly measure the (electron) temperature at $\approx 2 R_{*}$ to be $\approx 1500 \mathrm{~K}$. This is within the range needed to cause the large changes in visual light as outlined in this paper. Thus, based on direct observational

evidence, the simplicity of the mechanism described above, and the ability of our models to reproduce robustly the essential characteristics of Mira light curves, we feel that the physical picture outlined here may resolve the long-standing problem of the large amplitude of the light curves of Mira variables.

We thank R. Kurucz for providing the TiO cross-section data and the AAVSO for the visual data on $\chi$ Cygni from their International Database, which comprises observations by variable star observers worldwide.

\section{REFERENCES}

Bertschinger, E. \& Chevalier, R. A. 1985, ApJ, 299, 167

Bessel, M. S., Brett, J. M., Scholz, M. \& Wood, P. R. 1989, A\&A, 213, 209 
Bowen, G. H. 1988, ApJ, 329, 299

Celis, S. L. 1978, A\&A, 63, 53

Danchi, W. C., Bester, M., Degiacomi, C. G., Greenhill, L. J. \& Townes, C. H. 1994, AJ, 107,1469

Diamond, P. J. \& Kemball, A. J. 1999, in IAU Symp. No. 191: Asymptotic Giant Branch Stars, eds. T. Le Bertre, A. Lèbre \& C. Waelkens, (ASP; Provo, UT), p. 195

Feast, M. W., Glass, I. S., Whitelock, P. A., \& Catchpole, R. M. 1989, MNRAS, 241, 375

Ferlet, R. \& Gillet, D 1984, A\&A, 133, L1

Haniff, C. A., Ghez, A. M., Gorham, P. W., Kulkarni, S. R., Matthews, K. \& Neugebauer, G. 1992, AJ, 103, 1662

Haniff, C. A., Scholz, M. \& Tuthill, P. G. 1995, MNRAS, 276, 640

Helling, Ch., Winters, J. M. \& Sedlmayr, E. 2000, A\&A, 358, 651

Höfner, S., Jorgensen, U. G. Loidl, R. \& Aringer B. 1998, A\&A, 340, 497

Hoffleit, D. 1997, J. AAVSO, 25, 115

Johnson H. L. 1952, ApJ, 116, 272

Jorgensen, U. G. 1994, A\&A, 284, 179

Karovska, M., Nisenson, P., Papaliolios, C. \& Boyle, R. P. 1991, ApJ, 374, L51

Keeley, D. A. 1970, ApJ, 161, 657

Labeyrie, A., Koechlin, L., Bonneau, D. \& Blazit, A. 1977, ApJ, 218, L75

Lockwood, G. W. \& Wing, R. F. 1971, ApJ, 169, 63

Lopez, B. et al. 1997, ApJ, 488, 807

Mattei, J. 2001 Observations from the AAVSO International Database, private communication.

Merrill, P. W. 1940, Spectra of Long-Period Variable Stars, (U. Chicago, Chicago)

Pease, F. G. 1931, Ergebn. Exakten Naturwiss., 10, 84 
Pettit, E. \& Nicholson, S. B. 1933, ApJ, 78, 320

Plez, B., Brett, J. M. \& Nortdlund, A. 1992, A\&A, 256, 551

Reid, M. J. 1976, ApJ, 207, 784

Reid, M. J. \& Dickinson, D. F. 1976, ApJ, 209, 505

Reid, M. J. \& Goldston, J. E. 2001, in preparation.

Reid, M. J. \& Menten, K. M. 1997, ApJ, 476, 327

Reid, M. J., Menten, K. M. \& Goldston, J. E. 2001, unpublished data.

Slutz, S. 1976, ApJ, 210, 750

Smak, J. 1964, ApJS, 9, 141

Tej, A., Chandrasekhar, T., Ashok, N. M., Ragland, S., Richichi, A. \& Stecklum, B. 1999, AJ, 117, 1857

Tuthill, P. G., Haniff, C. A. \& Baldwin, J. E. 1999, MNRAS, 306, 353

Wildt, R. 1939, ApJ, 89, 295

Winters, J. M., Fleischer, A. J., Gauger, A. \& Sedlmayr, E. 1994, A\&A, 290, 623

Woitke, P., Helling, Ch., Winters, J. M. \& Jeong, K. S. 1999, A\&A, 348, L17

Wood, P. R. 1979, ApJ, 227, 220

Young, J. S. et al. 2000, MNRAS, 318, 381 


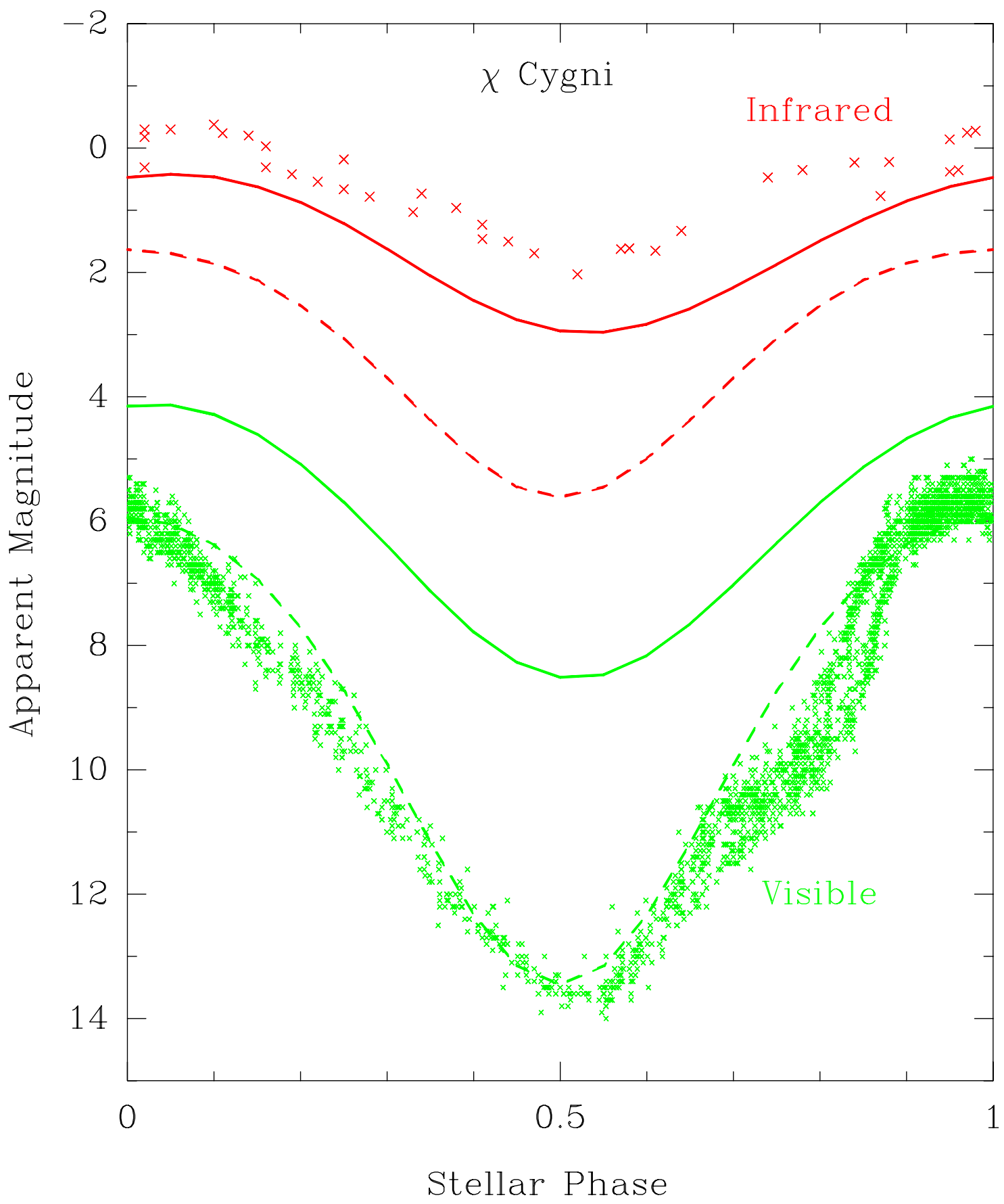

Fig. 1.- Observed infrared (red crosses), and visible (green crosses) light curves for the Mira variable $\chi$ Cygni. (See text for references.) Solid lines are for a black-body model in which the temperature and radius of the star vary as determined by Pettit \& Nicholson (1933). Dashed lines are for a black-body with a very large change in temperature: from $2000 \mathrm{~K}$ at maximum to $1240 \mathrm{~K}$ at minimum light. Note that black-body models cannot simultaneously fit the visible and infrared light curves. 


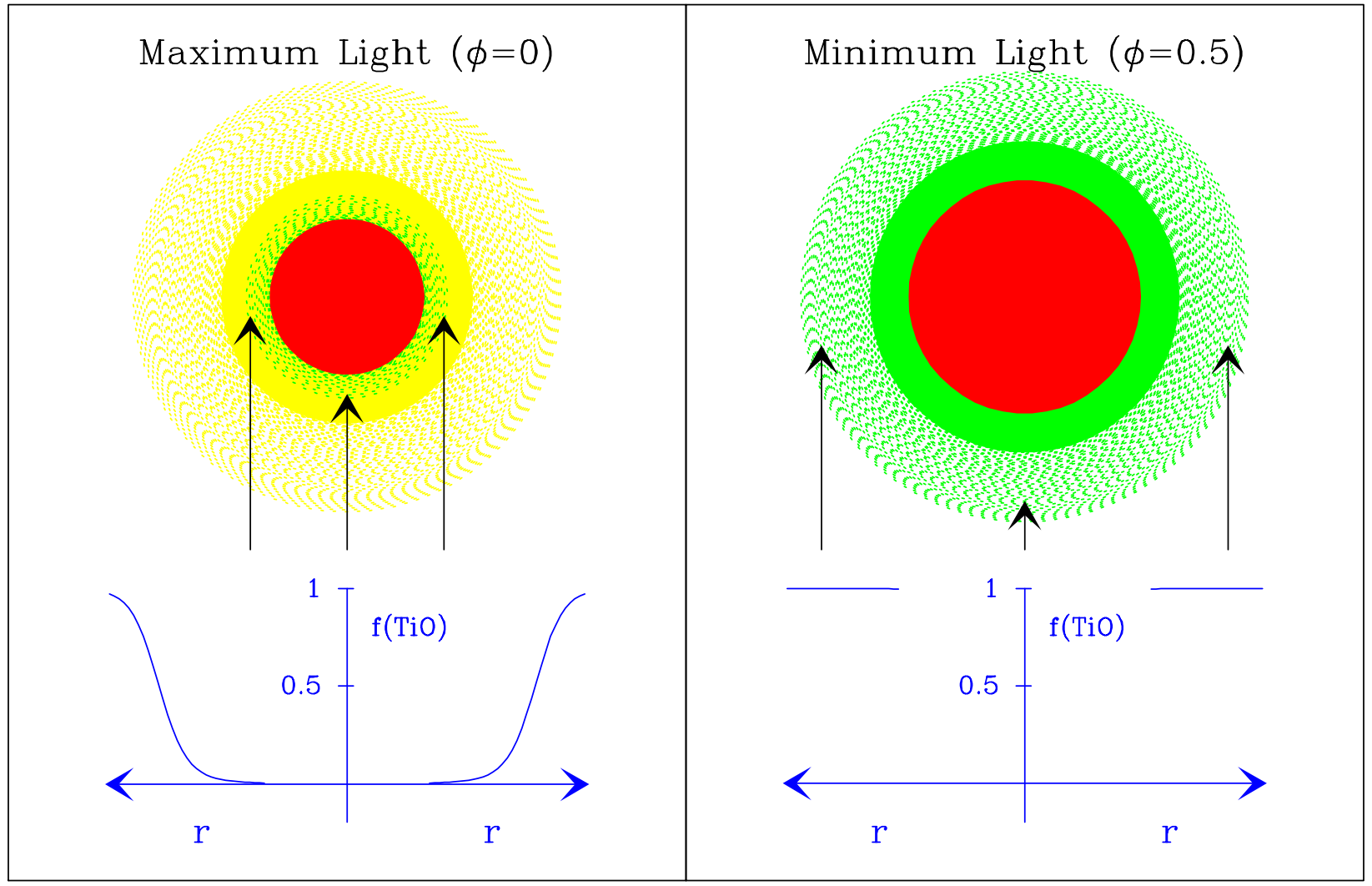

Fig. 2.- A schematic depiction of the change in visual appearance of a Mira variable star at maximum (left-hand panel) and minimum (right-hand panel) light. The star, shown in red, is smaller and hotter at maximum light than at minimum light. At maximum light, the extended atmosphere of the star (shown as yellow) is partially transparent at visual wavelengths, and one sees almost down to the stellar surface (indicated with arrows). Near minimum light, the temperature of the star has declined and metallic oxides, such as $\mathrm{TiO}$ (shown as green), form throughout the extended atmosphere. The fraction of $\mathrm{Ti}$ in $\mathrm{TiO}$, $\mathrm{f}(\mathrm{TiO})$, as a function of radius is plotted in blue. Near minimum light, TiO forms with sufficient density at a radius of $\approx 1.8 R_{*}$ to become opaque to visible light. At this radius, the temperature can be very low, and almost all radiation is in the infrared. Since little visible light emerges, the star can almost disappear to the human eye. 

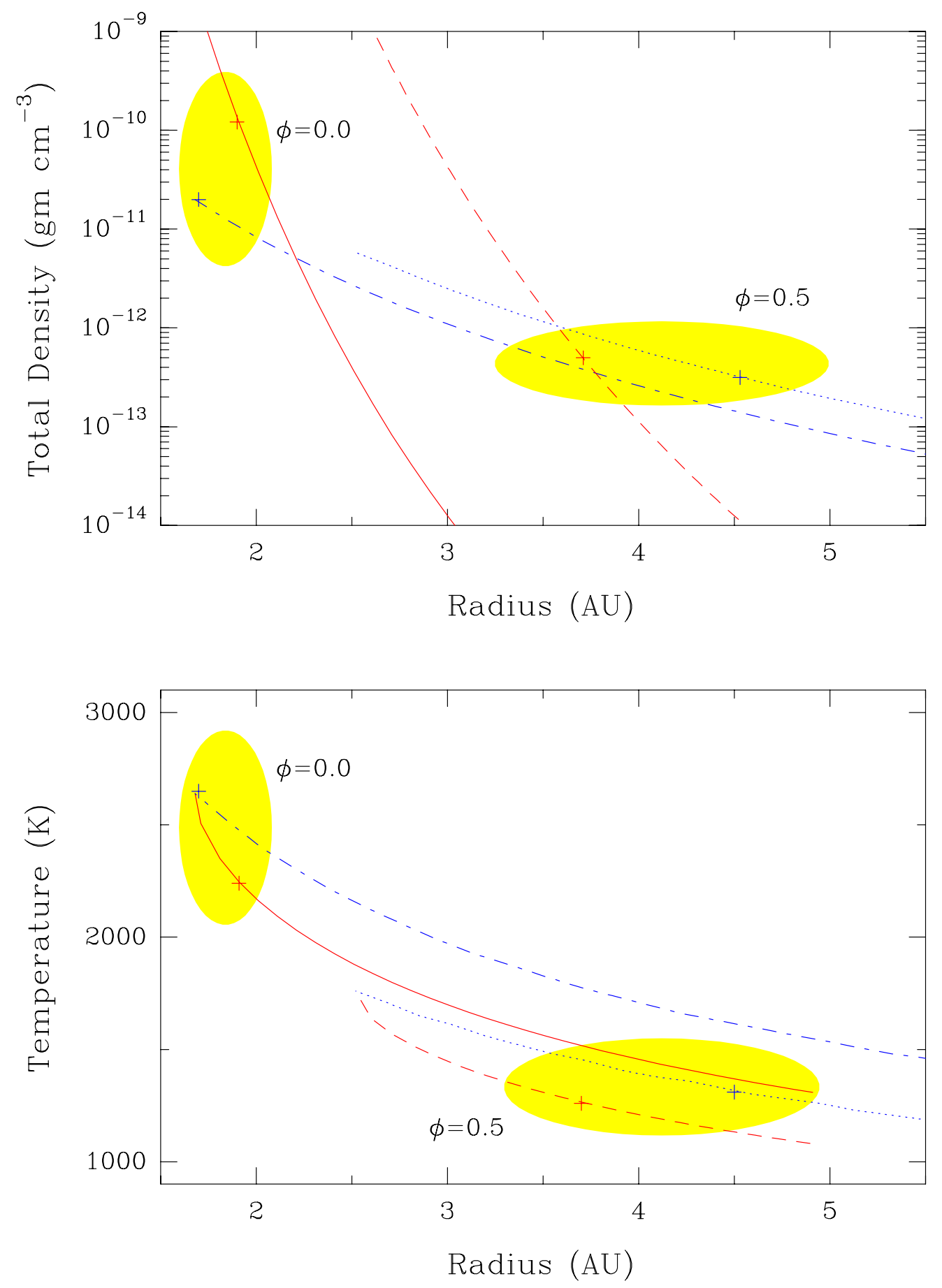

Fig. 3.- Models of density (upper panel) and temperature (lower panel) versus radius in the extended stellar photosphere of $\chi$ Cygni. The red solid and dashed lines refer to a hydrostatic model at maximum and minimum visible light. The blue dash-dotted and dotted lines refer to an alternative, power-law, model at maximum and minimum visible light. The yellow ellipses, labeled with visual phase $(\phi)$, indicate roughly the regions of parameter space that are likely to yield visual and infrared magnitudes similar to those observed. Pluses $(+)$ indicate radii at which the visual optical depth reaches unity in the models. 


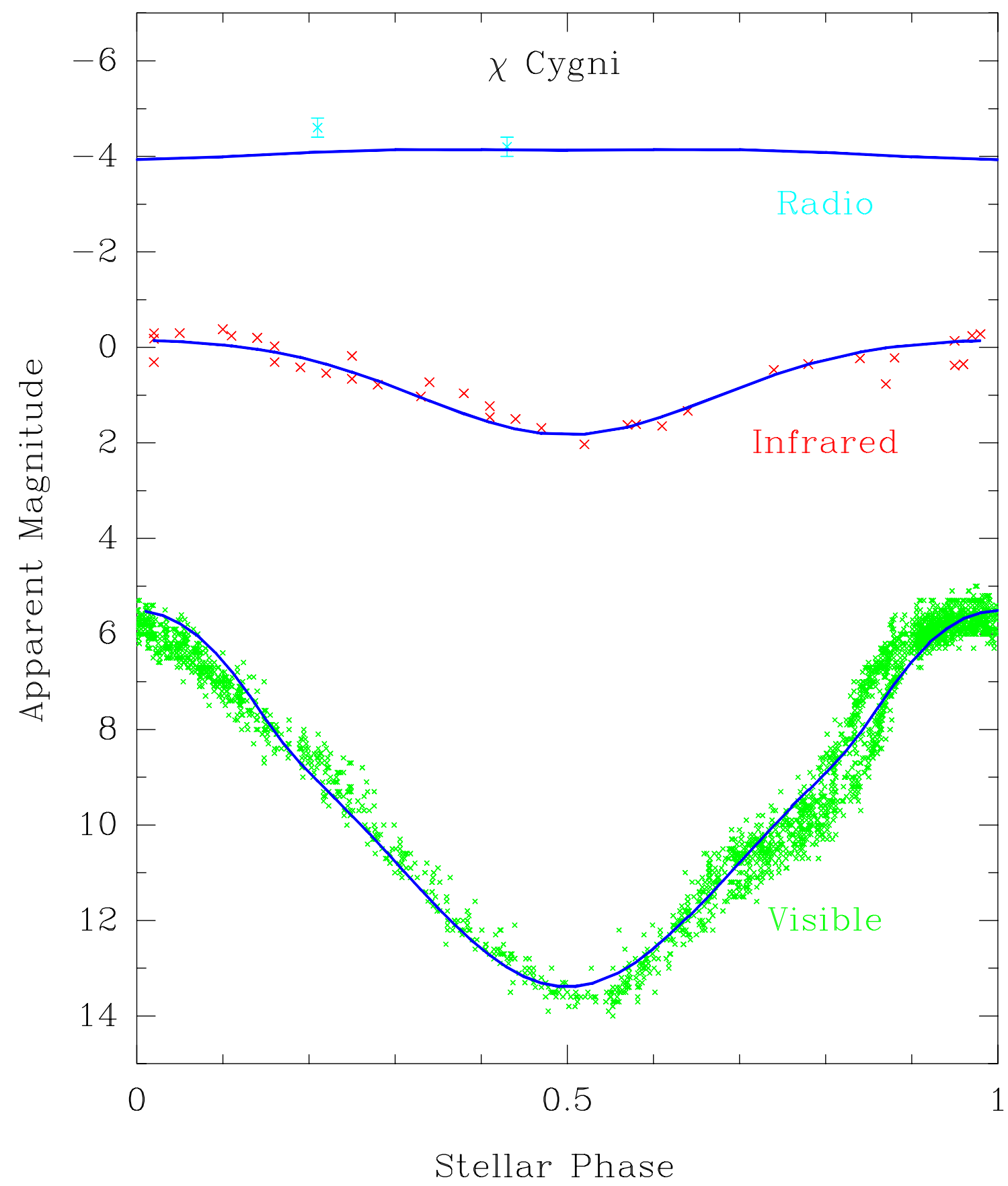

Fig. 4.- Observed radio (cyan), infrared (red), and visible (green) light curves for the Mira variable $\chi$ Cygni. (See text for references.) Solid blue lines are from a simple model of an oxygen-rich Mira variable with an extended atmosphere. Near stellar phase of 0.5 , molecular absorption, mostly by $\mathrm{TiO}$, enlarges the apparent visible size of the star to nearly $2 R_{*}$. The low atmospheric temperatures $(\approx 1400 \mathrm{~K})$ at this radius result in an $\approx 8$ magnitude decrease in visible light compared to maximum light. The infrared data are insensitive to molecular absorption and respond mostly to the central pulsating star. The radio emission is sensitive to the free electron density, and high radio opacity, owing to $\mathrm{H}^{-}$free-free interactions, occurs near $\approx 2 R_{*}$ throughout the stellar cycle. 